\title{
Towards the Ultimate Construction Site Sensor ${ }^{1}$
}

\author{
by
}

\author{
William C. Stone ${ }^{2}$ and Maris Juberts ${ }^{3}$
}

\begin{abstract}
The NIST Construction Metrology and Automation Group (CMAG), in cooperation with the NIST Intelligent Systems Division (ISD), is developing performance metrics and researching issues related to the design and development of a "Next Generation LADAR" sensor that will enable general automation in structured and unstructured environments. This paper quickly reviews the basic physics and implementation of various LADAR technologies, describes the problems associated with available "off-the-shelf" LADAR systems, summarizes State-of-the-Art work underway around the world, and elaborates on general directions that advanced research in this area of sensor design will take in the coming years and its likely impact on construction automation.
\end{abstract}

KEYWORDS: AM-modulation, construction automation, FM-modulation, laser radar, multiple returns, phantom points, pulse time-of-flight, range image sensors

\subsection{INTRODUCTION}

Laser Detection and Ranging (LADAR) is currently poised to become the ubiquitous $3 \mathrm{D}$ spatial measurement tool in many disciplines. Initially used for remote sensing and aerial surveying, LADAR applications now include reverse engineering (3D models), ground surveys, automated process control, target recognition, and autonomous machinery guidance and collision avoidance to name just a few. Efforts are currently underway at NIST to develop national artifact-traceable LADAR calibration facilities; to develop rapid, LADAR-based long range autoID systems; and to establish the scientific and engineering underpinning needed to develop miniature, high resolution next-generation LADAR systems.

The power of LADAR lies in the inherent 3D nature of the data it produces, namely spatial coordinates associated with each pixel in a so- called "range image" acquired by the device. A range image is effectively a spherically acquired $(\mathrm{r}, \theta, \varphi)$ dataset mapped to a 2D matrix, or "frame." LADAR frames are frequently presented as false color depth images. Additional data, including reflectance intensity associated with each pixel and multi-spectral intensity information, are commonly available. Color reflectance intensity (as opposed to active illumination frequency-specific reflected intensity) is obtained from co-boresighted RGB CCD sensors. Such a wealth of information can be rapidly segmented for use by a wide variety of real-time systems for machine control and post-processed for such metrology applications as as-built geometry checking for buildings and other civil infrastructure. This said, why are we not seeing LADAR systems on every construction site? The reasons most frequently cited are: slow speed of operation, bulky, high cost, and widely varying accuracy that presently lacks standardized calibration metrics. There are other related issues such as

1 Official contribution of the National Institute of Standards and Technology (NIST); not subject to copyright in the United States.

2 NIST, BFRL, Construction Metrology and Automation Group, Mail Stop 8611, Gaithersburg, MD 20899-8611; william.stone@nist.gov.

3 NIST, MEL, Systems Integration Group, Mail Stop 8230, Gaithersburg, MD 20899-8230; maris.juberts@nist.gov 
methods for processing the data (both real-time and offline) but that is the subject of another paper. Frequently the performance of a LADAR is defined by the following metrics:

- Maximum permissible illumination power

- Sensor horizontal Field of View (FOV)

- Sensor vertical FOV

- Wavelength of optical source

- Maximum distance to be measured

- Measurement time

- Measurement resolution (depth)

- Measurement resolution (angular)

- Range Measurement accuracy

To these one frequently must consider:

- Intensity of background (passive) illumination

- Color temperature of the (passive) background

- Target reflectivity (texture, color, specularity)

- Angle of beam incidence on the object

- Overall size (volume) of the sensor

- Manufactured cost of the sensor

At the conclusion of this paper we will present a set of design criteria we feel are representative of those needed to achieve ubiquitous use of LADAR sensing for construction operations. We will also comment on the research needed to achieve a physical sensor meeting such criteria.

\subsection{LADAR PHYSICS SUMMARY}

\subsection{Pulse Time-of-Flight (TOF)}

Figure 1 shows a "family tree" of LADAR devices that have at one time or another been built to operate at optical and near-optical wavelengths. References 1 and 2 provide useful indepth discussions on many of these devices. The simplest of the concepts uses pulse time-of-flight (TOF), as illustrated in Figure 2. An illumination pulse is generated, frequently by means of a NdYAG microchip laser, and the time of this event is made available to a timing circuit. The beam traverses a distance equal to $2 d$ and arrives at a photonic detector in time:

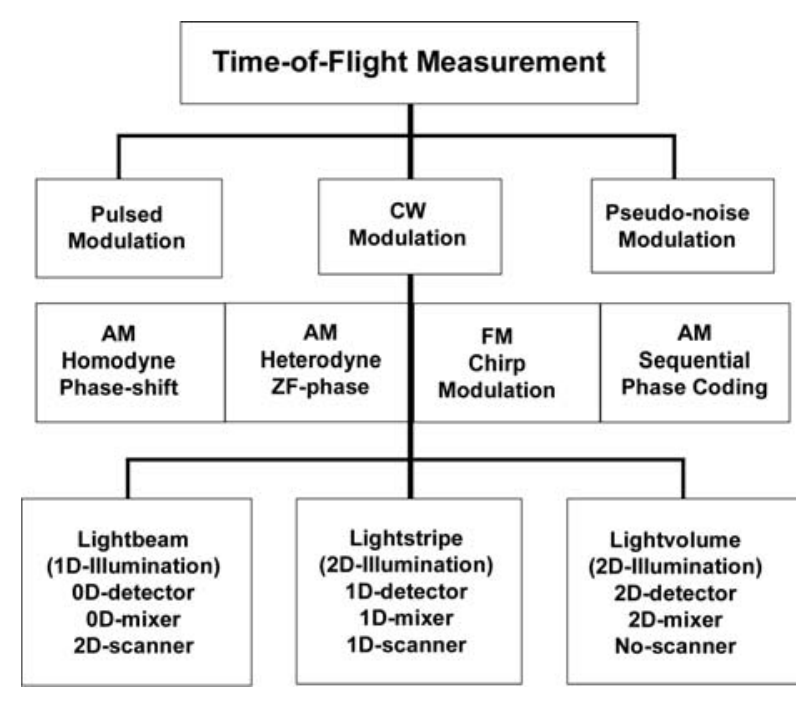

Figure 1: "Family Tree" of optical and near-optical wavelength time-of-flight range measurement devices.

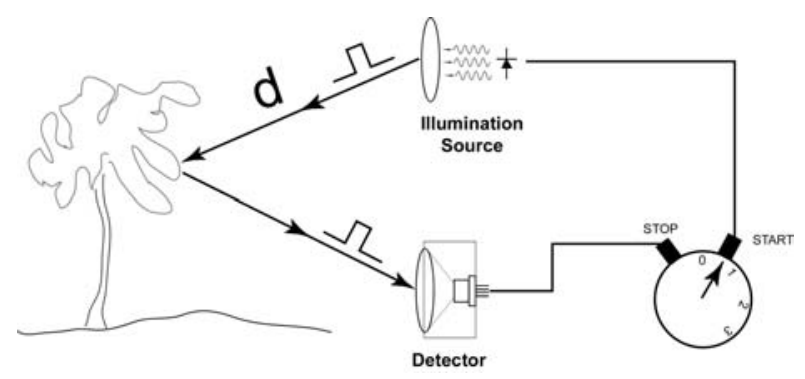

Figure 2: Fundamental physics of pure "pulsed" timeof-flight. Key performance metrics are synchronization precision of pulse initiation between the source and detector, pulse width, and detector bandwidth.

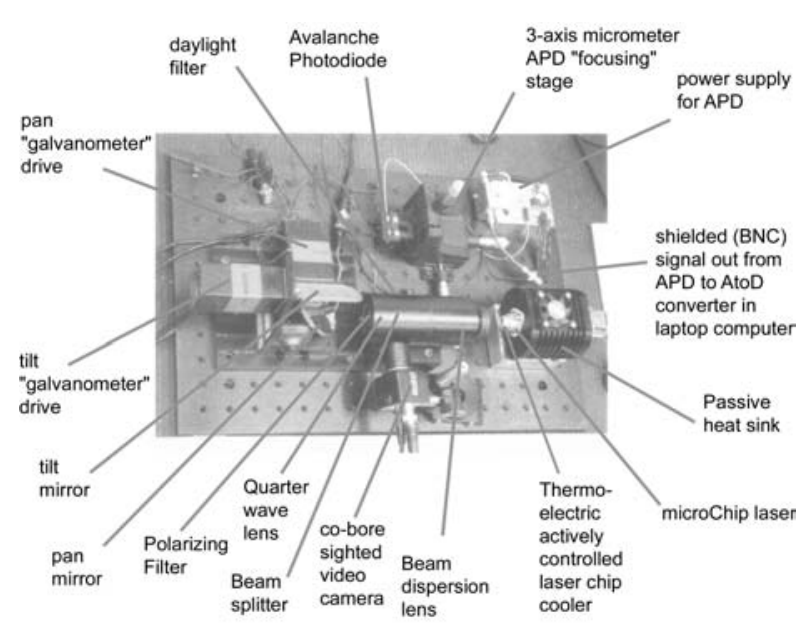

Figure 3: Typical physical implementation of a pure pulsed time-of-flight LADAR Scale is approximately $300 \mathrm{~mm}$ long by $150 \mathrm{~mm}$ wide. 


$$
t=\frac{2 d}{c}
$$

where $\mathrm{c}=$ speed of light. A clock time of $1 \mathrm{~ns}$ represents a $300 \mathrm{~mm}$ round-trip flight or an absolute range of $150 \mathrm{~mm}$. One can immediately see that an extremely accurate clock is required to achieve a level of accuracy sufficient for autonomous fabrication. Figure 3 shows a typical physical implementation of a pulse TOF LADAR [8]. The microchip laser generates $1 \mathrm{~ns}$ pulses at a rate of $10 \mathrm{kHz}$, producing an unambiguous range interval of $15 \mathrm{~km}$. The signals are detected by an avalanche photodiode (APD). In this particular implementation an optical beam splitter is used to divert a portion of the source signal to the APD, thus providing the "start timer"mark for range determination. The accuracy of such systems depends on a number of factors including the pulse width, detector electronics bandwidth, and the processor implementation. If the APD bandwidth is $2 \mathrm{GHz}$ and the analog-to-digital readout (also known as ROIC) is matched, then the timing "bin" width is approximately $0.5 \mathrm{~ns}$, but since that is round-trip, the range bin accuracy is thus $75 \mathrm{~mm}$. This " 0 $\mathrm{D}$ " ranging system is then scanned in $2 \mathrm{D}$ using electromechanically steered mirror systems.

\subsubsection{Limitations of Pulse TOF}

Thus far we have made the assumption that all of the photons that are generated hit one specific object and are reflected back to the detector in a narrowly discriminating beam yielding one range measurement per pixel. This is not the case as shown in Figure 4. Due to imperfect optics and atmospheric dispersion the source illumination beam (pulse) expands with range; good industrial LADARS that have achieved near-diffraction limited optics have beam dispersion angles of around $0.2 \mathrm{mrad}$. Even so, this produces a finite beam diameter at $100 \mathrm{~m}$ of around $20 \mathrm{~mm}$. This has interesting physical consequences. Because the un-ambiguous range of the device shown in Figure 3 is on the order of $15 \mathrm{~km}$, one receives, in time, responses from photons from the illumination pulse arriving at different times related to
Uncompensated MicroChip Laser Beam Divergence

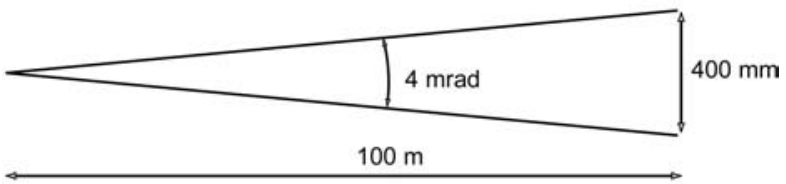

With Diffraction-Limited Optics

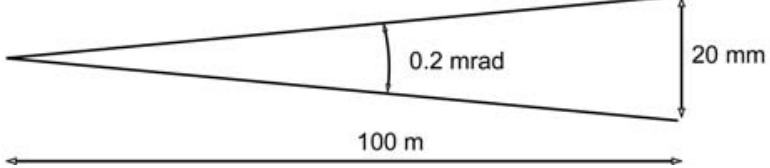

Figure 4: Source beam divergence variance and its effect on absolute beam diameter at $100 \mathrm{~m}$ range.

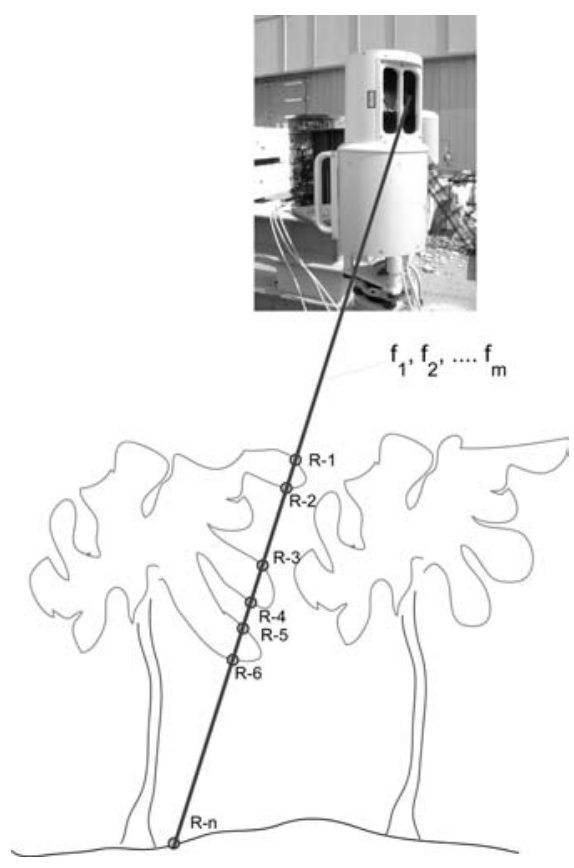

Figure 5: Due to beam divergence photons associated with a single "pixel" in a LADAR frame may represent significantly differing range data.

the different objects they hit within the cone of the dispersed beam. Figure 5 illustrates this point. A single "pixel" in the LADAR frame will in fact have multiple valid ranges. Figure 6 shows a time-domain response out to a range of approximately $120 \mathrm{~m}$. Any strong return above the noise threshold represents a valid object detection. Thus, one could store not one value, but a vector of values, for each pixel. Some LADARs now being developed illuminate at more than one wavelength. The response at each 


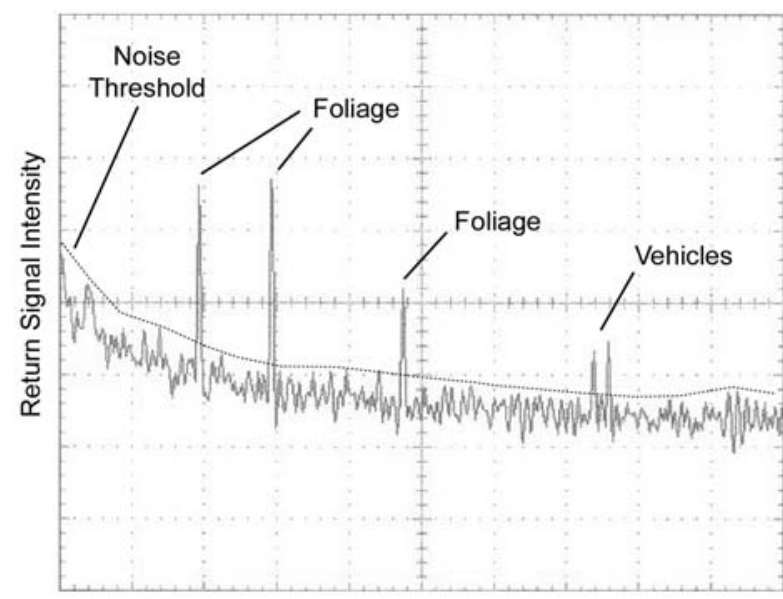

Time from Source Pulse Trigger ( $\mathrm{ns}$, full frame width $=400 \mathrm{~ns}$ )

Figure 6: Full time-domain response for a 1ns pulse. Response was captured in 801 "range bins." The time-width of each bin was $0.5 \mathrm{~ns}$, or $150 \mathrm{~mm}$.

pixel can then be represented by a matrix with $n$ returns per source frequency f:

$$
\left[\begin{array}{ccccc}
R_{1, f_{1}} & R_{1, f_{2}} & R_{1, f_{3}} & \ldots & R_{1, f_{m}} \\
R_{2, f_{1}} & \cdots & \cdots & \ldots & \cdots \\
R_{3, f_{1}} & \cdots & \ldots & \ldots & \ldots \\
\cdots & \ldots & \ldots & \ldots & \ldots \\
R_{n, f_{1}} & \cdots & \ldots & \ldots & R_{n, f_{m}}
\end{array}\right]
$$

At present, no commercial pulse TOF LADAR provides the user with this kind of pixel response matrix (or even a single frequency time domain vector). Instead, it is common to average earlier arrivals that have strong $\mathrm{S} / \mathrm{N}$ (signal-to-noise) ratios and report that as a single range per pixel. The results of this averaging are shown in Figure 7, where non-existent "phantom" points become part of the point cloud data set. The point here is that each of the valid returns shown in Figure 6 represent usable engineering information that is presently not available. Pulse TOF systems are limited in their accuracy not only by the bandwidth of the detector (currently pegged at around $2 \mathrm{GHz}$, although current research in fiber optic tele-communications is pushing this higher), but also by the pulse width of the source illumination, since edge detection is enhanced by a shorter, sharper pulse. The shortest pulse source cur-

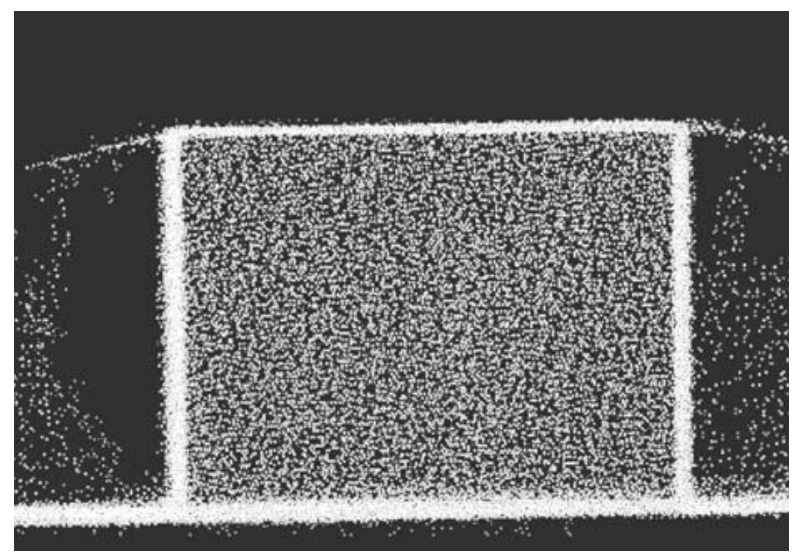

Figure 7: "Phantom" points (sloping ledges leading diagonally downward from the top) generated during scans of a rectangular box using single-point-per-pixel reporting from an industrial pulse TOF LADAR. Averaging of multiple returns (see Figure 6) to provide a single range per pixel leads to the erroneous reported locations.

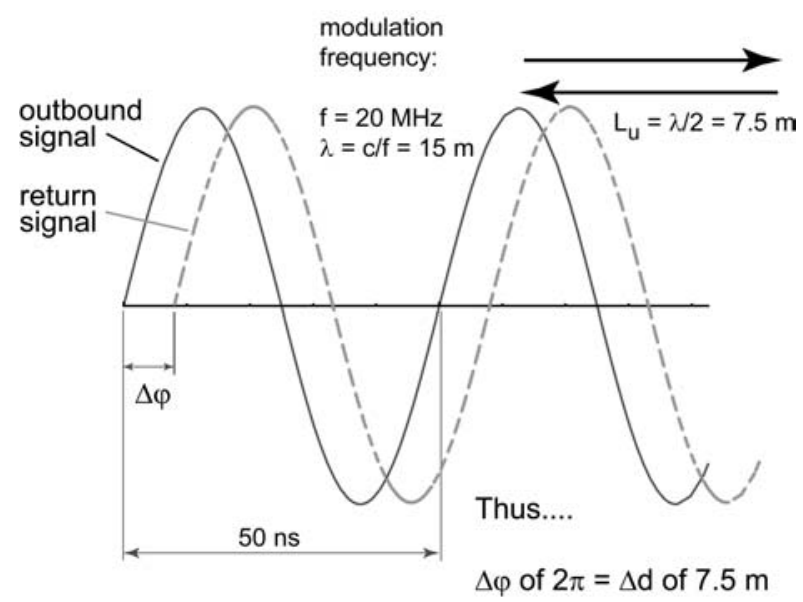

Figure 8: Phase-based determination of time-of-flight. Primary modulation frequency establishes the maximum unambiguous range for the device.

rently used in LADAR devices is 250 ps [5].

\subsection{AM Homodyne Phase Modulation}

One way to beat the "brute force" approach to time-of-flight accuracy is through the use of phase detection. This concept is illustrated in Figure 8. If the source is modulated at a single sinusoidal frequency, $f$, then a phase shift of

$$
\Delta \phi=2 \pi f T=2 \pi f \frac{L}{c}
$$


will be observed between the transmitted and received signal. Since the range, $R$, is equal to half the round-trip distance, $\mathrm{L}$,

$$
R=\frac{\Delta \phi c}{4 \pi f}
$$

The unambiguous range resolution is directly proportional to the source modulation frequency, $\mathrm{f}$, while the accuracy is directly proportional to the signal-to-noise ratio. The two are directly related; the tighter the unambiguous range the finer can be parsed the phase difference, thus improving the accuracy. For the case shown in Figure 8, the unambiguous range is half the wavelength at $\mathrm{f}=20 \mathrm{MHz}$, or $7.5 \mathrm{~m}$. Signal-tonoise ratio can be improved by integration over many cycles, but at the cost of raw throughput (frame rate).

Range determination using phase measurement is adapted from earlier work in radar in which the source and received signals are mixed and the phase, amplitude, and offset of the resulting signal are determined through Fourier theory. If the resulting signal is sampled at four intervals separated by phase angles of $\pi / 2$, it can be shown $[2,6]$ that the phaseshift, $\Delta \phi$, is given by

$$
\Delta \phi=\arctan \left[-\frac{A_{1}-A_{3}}{A_{0}-A_{2}}\right]
$$

where $A_{0}, A_{1}, A_{2}, A_{3}$ represent the integration of the mixed signal over the intervals shown in Figure 8 . The trick is in the formation of the

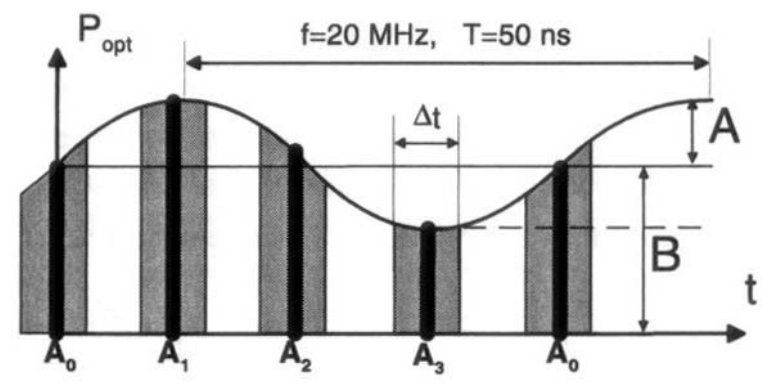

Figure 8: In a phase-based solution the transmitted and received signals are mixed. Discrete Fourier Transform (DFT) theory allows extraction of the phase, amplitude, and offset by sampling four points at an interval of $\pi / 2$ along the resulting waveform.

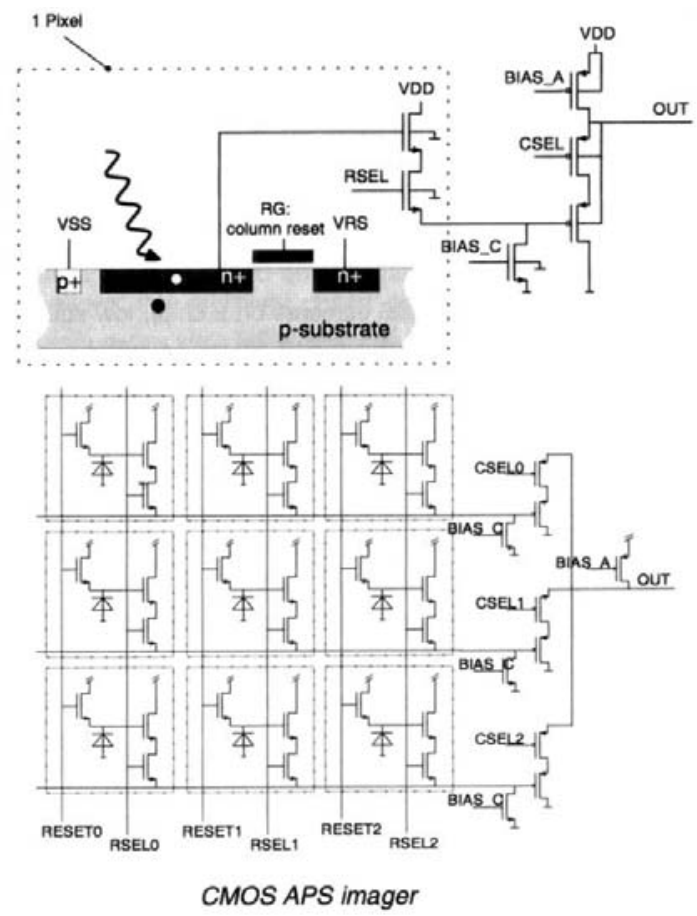

Figure 9: Example of an "Active Pixel Sensor," one of many versions of a Focal Plane Array (FPA) that can be used to simultaneously sample hundreds to thousands of pixels. FPAs form the core of "flash" LADAR and can utilize either phase or pure time-offlight ranging.

mixed signal and in the integrations to be performed. One of the more clever solutions to this problem makes use of so-called photonic mixing (Figure 9) in which a standard CMOS photo diode is reverse biased by the same frequency source that modulates the transmitted illumination signal. This diode responds directly to the mixed signal consisting of the modulated bias and the incoming photo electrons. Timing circuitry dumps the white portions of the curve in Figure 8 while summing (through a capacitor) the regions $A_{0}, A_{1}, A_{2}, A_{3}$ and selectively storing those values. It is possible to implement such detectors in 2D arrays in the form of Focal Plane Arrays (FPAs) as shown in Figure 9. Individual pixels within a LADAR frame are mapped (optically) to pixels on the FPA. Similar FPAs can be constructed to work on the pulse TOF principle. Both approaches are referred to as flash LADAR. 


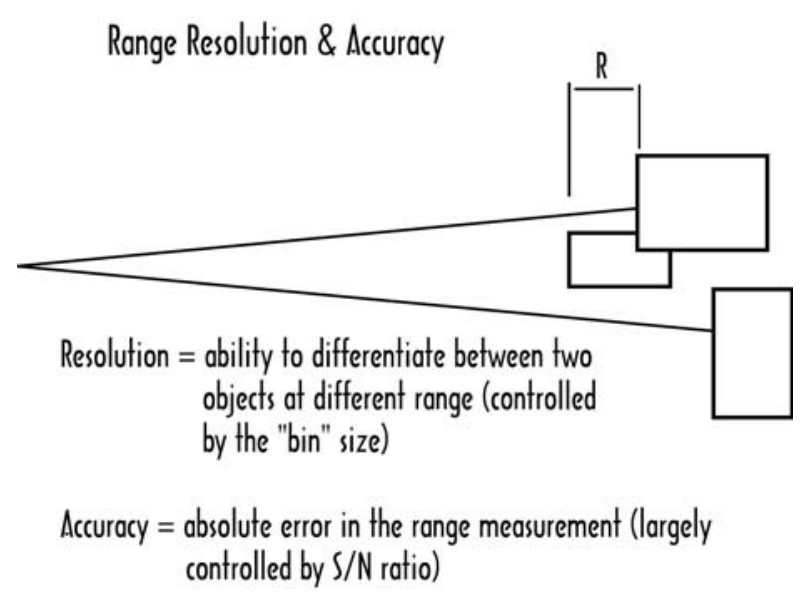

Figure 10: Due to beam divergence and varying reflectivity of target surfaces, both accuracy and resolution are affected for all types of LADAR, but particularly for AM-homodyne class devices which in effect integrate the reflected photons from all surfaces that are illuminated, thereby producing an erroneous average range.

\subsubsection{Limitations of AM Phase Measurements}

Pure homodyne (single frequency) LADARs suffer from two significant limitations. As the modulation frequency is increased, thereby improving potential accuracy, the un-ambiguous range is reduced -- leading to aliasing or false targets. A solution to this problem is to use multiple frequencies [4] in which a lower frequency signal is used to establish an un-ambiguous interval over a longer distance within which the higher frequency response is located. The mathematics for solving this approach are not significantly more complicated than for the pure homodyne solution, but it has yet to be implemented in silicon. A more serious problem with phase-based measurement is illustrated in Figure 10. For the same reasons that multiple returns are received in pulse TOF systems, the integration response of the photonically mixed signal includes the energy reflected from all the surfaces shown in Figure 10 , regardless of their different ranges, as long as they are within the illumination cone of the dispersed source beam. Thus, the range reported is an average of the objects within that pixel, leading to the same type of phantom points described earlier.

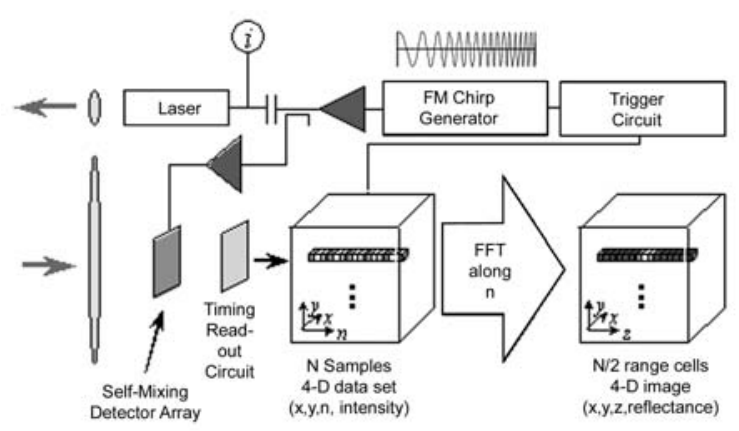

Figure 11: Block diagram for an experimental FMCW LADAR. This design overcomes range averaging issues with AM-CW system [7].

\subsection{FM-CW Modulation}

It is possible to recover multiple ranges per pixel in a frequency modulated LADAR by producing a very specifically-shaped source pulse, known as a chirp. The theory for such an approach has been around since the advent of radar [7] but only recently has been adapted to optical systems. In a chirp pulse the frequency is varied linearly with time; typically over the range of $200 \mathrm{MHz}$ to $600 \mathrm{MHz}$ with a frequency response of $800 \mathrm{MHz}$ or more. The range resolution (per pixel) in such a device is given as

$$
\Delta R=\frac{c}{2 \Delta f}
$$

in which $\Delta \phi$ is the chirp frequency step size. The detector in Figure 11 consists of a photonic FPA mixer and CMOS read out circuit. The result is a frequency-domain signal (an integrated intensity for each of $\mathrm{n}$ frequencies contained in the chirp bandwidth). This signal must be subsequently processed, using an FFT, to obtain a time domain response. Nyquist sampling criteria increases the range bin size by a factor of 2 .

The chief limitations of the FM-CW LADAR approach are the need for additional front end hardware (complicated chirp-generation electronics that add both cost and an additional level of noise), the frequency response of the laser source (which may be bandwidth limited); and the manifold increased mathematical post-processing that is involved. 


\subsection{IDEAL LADAR PERFORMANCE}

NIST has for a number of years conducted research in autonomous robotic platforms and machinery and has reached a consensus regarding the required performance of a "vision" system needed for effective control. A LADAR meeting these criteria would have the following attributes:

Illumination Source: eyesafe

Field of View (FOV): $60^{\circ} \times 60^{\circ}$

Range resolution: $1 \mathrm{~mm} @<15 \mathrm{~m}$

$$
3 \mathrm{~mm} @<100 \mathrm{~m}
$$

Angular resolution: $<0.03^{\circ}$

Frame rate: $>10 \mathrm{~Hz}$

Size: coffee cup

Cost: $<\$ 1000$ US

Some of these criteria can be met by existing systems but most cannot. Frequently there is a tradeoff between speed and accuracy. 2D frames can be created using high resolution laser rangers by a method known as "scanning" in which a single-degree-of-freedom laser radar is mechanically swept over the scene using either encoderequipped pan/tilt servos or a rotating mirror combined with either a pan or tilt servo. Because of this mechanical reliance, however, these systems have inherent speed and accuracy limitations associated with the servos and their encoders. New work in FPA design shows promise for both improving range resolution as well as speed. And only this latter approach shows promise for the miniaturization needed to achieve the last two criteria listed above. The FOV and angular resolutions listed above translate to a 2048 x 2048 pixel FPA. The largest range-imaging FPA yet to be demonstrated successfully is $25 \times 64$, although several labs have $128 \times 128$ arrays under development. There are other significant issues: to illuminate a large FOV requires considerable laser power, possibly making the output unsafe (eyesafe) at most of the compatible wavelengths. Parallel FPAs or MEMs-based steering provide possible solutions.

\subsection{Conclusions and Avenues for Research}

The joint NIST effort to develop a Next Generation LADAR (NGL) for autonomous machine control and construction metrology has identified four key areas for research needed to achieve a functioning sensor with the above specifications:

- Ultra-fast Chip-Level Laser technology: build and test compact pulsed femtosecond $\left(\sim 10^{-13} \mathrm{~s}\right)$ coherent laser sources. The performance, power needed, source-detector cross-talk and ranging accuracy of prototype lasers and APD receivers will be characterized at NIST. It will be determined whether nonlinear quantum dot saturable absorbers can be implemented in micro-laser cavities to achieve shortest possible pulse duration. Initial efforts will be to experimentally determine if extensions to known micro-chip laser systems can achieve desired femtosecond performance followed by work on candidate new designs.

- Ultra-precise Chip-Level Time and Frequency Standards: Investigate development of MEMs/CMOS-based manufacture of on-chip, high accuracy time and frequency standards and phase correlators. Investigate development of candidate designs for CMOS implementation of the best timing and frequency designs and ultimately to the fabrication of test articles. Refine designs of an on-chip timing and frequency standard that could be readily integrated with a candidate APD/FPA for testing with either the chip light source developed in task 1 or by means of external femtosecond laser sources.

- Fast Beam Steering: There are several different technologies available for fast beam steering. The most popular of these are acousto-optic, tilting mirrors and electro-holography. Miniature beam steering devices are being investigated at NIST based on these technologies. The tilting mirror technology has received special attention recently, because MEMS-size mirror arrays have been built. Acousto-optic devices use acoustic waves propagating in a variety of optic materials to control the refractive index of the material and thus the angle of the output light beam. The 
electro-holography technology involves the writing of Bragg grating holograms of specific wavelengths on photo-refractive crystals. NIST is evaluating these beam steering technologies and will select the most promising of them for prototype devices.

- Systems Integration and Performance Analysis: Early analysis here has focused on the advantages and disadvantages of direct TOF versus phase correlation methods for range determination within the context of systems capable of being fabricated using MEMs and CMOS technology combined with InGaAs / InP detectors that are either bump or bridge-bonded to the read out circuit. This effort is also investigating issues and techniques for integrating a single channel micro-steerable LADAR. Computer simulations are being developed to evaluate the feasibility of miniaturization, operation in various environments, sensor detection limits, and performance characteristics - distance, accuracy, and speed.

The results of this research in the coming years will prove the feasibility of building the ultimate construction site sensor.

\subsection{REFERENCES}

1. Besl, P.J., "Active, Optical Range Imaging Sensors," Machine Vision and Applications (1988) 1:127-152.

2. Lange, R., "3D Time-of-Flight Distance Measurement with Custom Solid-State Image Sensors in CMOS/CCD-Technology," Doctoral Dissertation, Dept. of Electrical Engineering and Computer Science at University of Siegen, Siegen, Germany, June 28, 2000.

3. Stettner, R., and Bailey, H.W., "3D Imaging Laser Radar," United States Patent 6,133,989, October 17, 2000.

4. Frohlich, C., Mettenleiter, M., Hartl, F., Dalton, G., and Hines, D., "Imaging Laser Radar for 3-D Modeling of Real World Environments," Sensor Review, Volume 20, No. 4, 2000, pp 273-281.
5. Aull, B.F. et al, "Geiger-Mode Avalanche Photodiodes for Three-Dimensional Imaging," Lincoln Laboratory Journal, Volume 13, Number $1,2001$.

6. Spirig, T., "Smart CCD/CMOS Based Image Sensors with Programmable, Real-Time, Temporal and Spatial Convolution Capabilities for Applications in Machine Vision and Optical Metrology," Doctoral Dissertation, Swiss Federal Institute of Technology, Zurich, 1997.

7. Stann, B.L., Ruff, W.C., and Sztankay, Z.G., "Intensity-Modulated Diode Laser Radar using Frequency-Modulation / Continuous-Wave Ranging Techniques," Optical Engineering, November 1996.

\section{Schilling, B.W., Barr, D.N., Templeton,} G.C., Mizerka, L.J., and Trussell, C.W., Multiple-return laser radar for three-dimensional imaging through obscurations, APPLIED

OPTICS, Vol. 41, No. 15 pp 2791-2799, May 2002 